\title{
Factores de riesgo asociados a padecer cáncer de piel de células basales y escamosas en el Mediterráneo
}

\author{
Marta Gil Barrachina \\ marta.gil.barrachina@gmail.com \\ Bárbara Hernando Fuster \\ Hernandb@uji.es \\ Conrado Martínez Cadenas \\ ccadenas@med.uji.es
}

\section{Resumen}

Introducción: Los cánceres de células basales $(\mathrm{BCC})$ y de células escamosas (SCC), clasificados como cánceres cutáneos no-melanoma, son los de mayor incidencia en todo el mundo. Los principales factores etiológicos asociados con la predisposición a cualquier tipo de cáncer cutáneo son ambientales (exposición solar) y genéticos (polimorfismos que determinan rasgos de pigmentación y de sensibilidad cutánea al sol). En este estudio caso-control, se analizaron diferentes variables de riesgo asociadas a tener cáncer cutáneo no-melanoma en una población mediterránea.

Métodos: De un total de 156 individuos españoles (56 con BCC o SCC y 100 controles) se extrajo una muestra de ADN genómico para analizar variantes genéticas asociadas a la pigmentación y la sensibilidad solar mediante secuenciación y genotipación. Además, se recogió información fenotípica mediante cuestionario.

Resultados: Los factores de riesgo asociados a BCC o SCC son tener mayor edad $\left(p=1,77 \times 10^{-47}\right)$, el pelo claro $\left(p=1,22 \times 10^{-3}\right)$, mayor número de nevus $(p=0,013)$ y lentigos solares $\left(p=3,77 \times 10^{-25}\right)$, así como exponerse habitualmente al sol $(p=0,019)$. Asimismo, los pacientes con cáncer cutáneo BCC o SCC suelen ser portadores de variantes $R$ en el gen $M C 1 R(p=0,019)$ y del alelo de riesgo en la variante rs4911442 del gen $\operatorname{ASIP}\left(p=8,70 \times 10^{-4}\right)$.

Conclusión: Este estudio confirma la importancia de la exposición solar de forma continuada en la aparición del cáncer cutáneo no-melanoma, ya que encontramos una fuerte correlación con la edad, con la historia de exposición solar y con la presencia de lesiones hiperpigmentadas benignas. Además, el riesgo esta significativamente influenciado por factores genéticos.

Palabras clave: cáncer de piel, carcinoma basocelular, carcinoma espinocelular, pigmentación, genética humana. 


\begin{abstract}
Introduction: Basal cell (BSC) and squamous cell (SSC) carcinomas, classified as non-melanoma skin cancers, are the most frequent worldwide. The main etiological factors associated with the predisposition to skin cancers are environmental (sun exposure) and genetic (polymorphisms determining pigmentation traits and skin sensitivity to the sun). In this case-control study, we analyzed several risk variables previously associated with non-melanoma skin cancers in a Mediterranean population.

Methods: A genomic DNA sample was collected from a total of 156 Spanish individuals (56 with BSC or SSC and 100 controls) in order to analyze several genetic variants previously associated with human pigmentation traits and skin sensitivity to sunlight. In addition, a standard questionnaire was used to collect phenotypic information.

Results: The risk factors associated with BSC or SSC in our population are being older $\left(p=1.77 \times 10^{-47}\right)$, having light hair $\left(p=1.22 \times 10^{-3}\right)$, having high nevi count $(p=0.013)$ and solar lentigines $\left(p=3.77 \times 10^{-25}\right)$, as well as being frequently exposed to sunlight $(p=0.019)$. Besides, individuals with BSC or SSC are usually carriers of R alleles in the MC1R gene $(p=0.019)$, as well as of the risk allele in the ASIP rs4911442 polymorphism $\left(p=8.70 \times 10^{-4}\right)$.

Conclusion: This study supports the key role of a continuous sunlight exposure in non-melanoma skin cancer development, since a strong correlation with age, the history of sun exposure and the presence of hyperpigmented benign lesions was observed. In addition, the risk seems to be significantly influenced by genetic factors.
\end{abstract}

Keywords: skin cancer, basal cell carcinoma, squamous cell carcinoma, pigmentation, human genetics.

\title{
Introducción
}

El cáncer de piel es una de las neoplasias más comunes en poblaciones de origen europeo y su incidencia está aumentando de forma exponencial cada año en casi todos los países del mundo. Según recientes estudios epidemiológicos, este incremento parece estar asociado a cambios en la forma de exponerse al sol, tanto en actividades relacionadas con el empleo como con el ocio (Apalla, Nashan, Weller y Castellsagué 2017).

Las neoplasias cutáneas resultan de la transformación de diversas células epidérmicas. El melanoma cutáneo se origina por la transformación de los melanocitos, mientras que el cáncer de piel no-melanoma surge de otras células epidérmicas, principalmente de los queratinocitos. Dependiendo de la capa epidérmica de origen, los cánceres de piel de tipo no-melanoma se subdividen en carcinomas de células basales (BCC) y carcinomas de células escamosas (SCC) (Scherer y Kumar 2010). A diferencia del melanoma, el cáncer de piel no-melanoma suele ser benigno y no suele presentar complicaciones clínicas, aunque en estados muy avanzados puede producir metástasis ganglionar.

El cáncer cutáneo es una patología multifactorial y los principales factores etiológicos asociados son tanto de carácter genético como ambiental. La radiación ultravioleta (UV) es el mayor riesgo ambiental para el desarrollo de cáncer de piel. La radiación UV es un agente mutagénico que tiene la capacidad de dañar la secuencia de ADN y, 
por lo tanto, se relaciona con la aparición de mutaciones somáticas que más tarde pueden ser clave en el proceso oncogénico (Scherer y Kumar 2010). En los carcinomas espinocelulares o basocelulares, las lesiones neoplásicas suelen aparecer en áreas del cuerpo expuestas de forma habitual al sol, tales como la cara, la nuca, el dorso de la mano y el antebrazo, y son más habituales en personas que trabajan al sol, como pescadores, agricultores o trabajadores de la construcción. Además, suelen aparecer en edades avanzadas, ya que el individuo ha estado expuesto durante más tiempo a la acción dañina de la luz solar.

El riesgo de cáncer de piel debido a la exposición solar esta favorecido por ciertos rasgos de pigmentación determinados genéticamente, como tener el color de piel claro, los ojos azules o verdes, el pelo rubio o rojo, así como tener muchas pecas o lunares. Además, una elevada sensibilidad de la piel a la exposición solar, con la aparición de quemaduras y la imposibilidad de bronceado, es también un indicador de una mayor susceptibilidad a cáncer de piel. Así pues, en individuos con una piel de color más oscuro que tienden a broncearse tras exponerse al sol, la incidencia de neoplasias cutáneas es menor debido al mayor nivel de eumelanina epidérmica sintetizada, la cual ejerce como factor fotoprotector capaz de absorber la radiación UV (Liu, Wen y Kayser 2013; Scherer y Kumar 2010)

Los rasgos de pigmentación son de carácter poligénico, es decir, están influenciados por muchísimos genes con efectos relativamente pequeños. Variantes genéticas en los genes que codifican proteínas implicadas en la vía de pigmentación han sido asociadas con características fenotípicas de pigmentación y sensibilidad cutánea a la radiación solar, pero también con el riesgo de diversos tipos de cáncer de piel. Así pues, alelos asociados a fenotipos de riesgo, como tener la piel clara, el pelo rubio o rojo, los ojos azules o verdes, lesiones hiperpigmentadas benignas y tendencia a quemarse en vez de broncearse tras exponerse al sol también se han correlacionado con el riesgo de cáncer cutáneo tanto de tipo melanoma como no-melanoma.

Se han descrito más de 100 genes asociados con la pigmentación humana, pero el gen del receptor de melanocortina $1(M C 1 R)$ es el más importante para determinar el riesgo de cáncer de piel. El gen $M C 1 R$ codifica para un receptor acoplado a proteína $G$ ubicado en la superficie de los melanocitos que, tras la unión a a-melanocortina ( $\alpha$ $\mathrm{MSH}$ ), activa varias cascadas de señalización que conducen a la síntesis de eumelanina, así como a la reparación del daño del ADN inducido por la radiación UV y al control de la proliferación celular y la apoptosis. El gen $M C 1 R$ es altamente polimórfico en poblaciones de origen europeo y se han descrito más de 100 variantes que afectan a la secuencia del receptor (Beaumont et al. 2005). Determinadas variantes en el gen $M C 1 R$, conocidas como variantes $\mathrm{R}$, anulan la función del receptor y limitan la síntesis de eumelanina. Como consecuencia, aumenta la ratio de feomelanina respecto a eumelanina en la epidermis. Así pues, las personas portadoras de variantes de pérdida de función en este receptor transmembrana suelen tener el pelo rojo, una mayor sensibilidad solar y pecas, así como un mayor riesgo de desarrollar cualquier tipo de cáncer cutáneo (Ibarrola-Villava et al. 2010; Matichard et al. 2004; Scherer y Kumar 2010).

Además del gen $M C 1 R$, existen otros genes claves en la síntesis de melanina que influyen en la pigmentación y la fotosensibilidad cutánea y, por lo tanto, en el riesgo de padecer cáncer cutáneo, como por ejemplo los genes ASIP, BNC2 e IRF4. El gen $A S I P$ codifica para el péptido de señalización agouti que antagoniza la función del receptor de transmembrana $M C 1 R$ e inhibe la síntesis de eumelanina, desplazando el equilibrio hacia la síntesis de feomelanina. Polimorfismos en IRF4 y BNC2 se han visto significativamente asociados con la respuesta por bronceado de la piel tras la exposición solar en poblaciones tanto del norte como del sur de Europa (Hernando 
et al. 2018a; Jacobs et al. 2015, 2013). Una vez sintetizada la melanina en los melanocitos, esta se transfiere a los queratinocitos para ser depositada en la superficie epidérmica. Así pues, además de la influencia del ratio feomenlanina/eumelanina, el color de la piel dependerá de la capacidad de transferencia del pigmento sintetizado a los queratinocitos (Wu y Hammer 2014).

Debido al incremento de la incidencia de cánceres cutáneos de células basales y escamosas en poblaciones caucásicas, incluidas las poblaciones del sur de Europa que presentan un fenotipo pigmentario de menor riesgo, este estudio tiene como objetivo esclarecer las variables de riesgo asociadas al desarrollo de un cáncer de piel de tipo no-melanoma en una población mediterránea de Castellón.

\section{Método}

Población de estudio y recogida de datos

En este estudio caso-control se incluyeron un total de 156 participantes voluntarios de origen español procedentes de la zona del Mediterráneo. Entre todos los participantes, 56 individuos padecían cáncer de piel no-melanoma (SCC o BCC). De cada uno de ellos se recogió una muestra de tejido cutáneo, la cual se obtuvo de las partes sobrantes de resecciones de piel realizadas para eliminar por escisión una lesión cutánea. Estas muestras se recogieron entre junio y agosto del 2017, por parte de los especialistas en dermatología del Hospital General de Castellón y del Hospital La Plana de Vila-real. Además, se incluyeron un total de 100 individuos libres de cáncer cutáneo. Para obtener ADN genómico de los individuos control se recogió una muestra de saliva utilizando una torunda estéril. Los individuos se reclutaron de forma consecutiva y debían ser mayores de edad. Debido a que se van a estudiar variantes genéticas en línea germinal es indiferente la procedencia de la muestra biológica recogida.

Se utilizó un cuestionario estandarizado para recopilar información de sexo, edad, lugar de nacimiento, rasgos de pigmentación (piel, cabello y color de ojos), historial de quemaduras solares en la infancia, sensibilidad de la piel a la luz solar (capacidad de bronceado frente a tendencia a quemadura), presencia de lesiones hiperpigmentadas benignas (lentigos solares y número de nevus), clasificación del tipo de piel según Fitzpatrick y hábitos de exposición al sol. Cada participante completó el cuestionario bajo la supervisión de un profesional. Todos los participantes del estudio fueron informados del estudio y dieron su consentimiento por escrito para ser incluidos en la investigación. Este proyecto fue aprobado por el Comité Ético de Investigación Clínica (CEIC) de ambos hospitales involucrados (Hospital General de Castellón y Hospital de La Plana de Vila-real) y por el Comité Deontológico de la Universitat Jaume I.

\section{Extracción del ADN genómico}

EI ADN genómico de los individuos con cáncer de piel se obtuvo de la muestra de tejido cutáneo resecada. Inmediatamente después de resecar el tejido, todas las biopsias cutáneas se sumergieron en la solución RNAlater para estabilizar el material biológico (Thermo Fisher Scientific, Walham, MA, EE. UU.). Una vez las muestras llegaron al laboratorio, las piezas de tejido fueron sumergidas en tiocinato de amonio al $3,8 \%$ (Sigma-Aldrich, St Louis, MO, EE. UU.) en solución salina tamponada con fosfato $(\mathrm{pH} 7,4)$ y se incubaron durante 3 horas a temperatura ambiente para poder separar la epidermis de la dermis. Las extracciones de ADN de todas las muestras 
biológicas se realizaron utilizando el QIAamp DNA Mini Kit (Qiagen, Hilden, Alemania), siguiendo las recomendaciones del fabricante.

\section{Análisis genéticos}

Se analizaron un total de 3 variantes genéticas localizadas en genes previamente asociados a rasgos de pigmentación humana y a la respuesta cutánea a la radiación solar: rs4911442 (localizado en el gen ASIP), rs2153271 (en el gen BNC2) y rs12203592 (en el gen IRF4). Todas las reacciones de genotipado se realizaron utilizando KASP SNP Genotyping Chemistry (LGC, Hoddesdon, Reino Unido). El análisis de la genotipación se llevó a cabo utilizando el equipo de PCR a tiempo real y utilizando el software SDS 2.1 (Applied Biosystems ${ }^{\mathrm{TM}}$ StepOne ${ }^{\mathrm{TM}}$ Real-Time PCR System Software) para el análisis de discriminación alélica. Las condiciones de PCR variaron según los requerimientos de cada sonda de genotipación, según las recomendaciones del fabricante. Como medida de control de calidad, se incluyó un control negativo y tres muestras con genotipo conocido (un heterocigoto, un homocigoto del alelo ancestral y un homocigoto del alelo derivado) por placa de 96 pocillos. Una vez obtenidos los resultados, los individuos fueron clasificados como homocigotos del alelo mayor, homocigotos del alelo menor o heterocigotos para cada uno de los polimorfismos genotipados.

El análisis de las variantes genéticas presentes en el gen $M C 1 R$ se realizó mediante una secuenciación completa de la región codificante del gen, ya que se trata de un gen altamente polimórfico, utilizando la tecnología Sanger (Ibarrola-Villava et al. 2010). Como medida de control de calidad, se incluyó una muestra con genotipo conocido (control positivo) y un pocillo sin muestra (control negativo) por placa de 96 pocillos. Las mutaciones no sinónimas detectadas en el gen $M C 1 R$ se clasificaron como $R$, si conducían a la pérdida completa de la función del receptor; como $r$, si provocaban una pérdida parcial de la función del receptor, o como $p$, si no afectaban a la función del receptor transmembrana (Hernando et al. 2018b). Los individuos fueron entonces clasificados como portadores o no de variantes de pérdida de función en el gen $M C 1 R$ (alelos R).

\section{Análisis estadísticos}

Inicialmente se realizó un análisis descriptivo de las variables estudiadas según si los individuos eran casos o controles, presentando los datos como media y desviación típica para las variables cuantitativas y como tamaño muestral y porcentaje para las variables categóricas. El test de Shapiro-Wilk fue utilizado para determinar si la variable principal del estudio seguía una distribución normal en nuestra población. El test chi-cuadrado se utilizó para comprobar si existían diferencias significativas en la distribución de las variables categóricas entre casos y controles. El análisis de varianza (ANOVA) de un factor se empleó para determinar la posible igualdad de medias de una variable cuantitativa entre casos y controles. Debido a la existencia de diferencias en la edad entre casos y controles, y para corregir la posible influencia de esta variable sobre las otras, los análisis estadísticos se repitieron teniendo en cuenta la edad mediante regresión logística bivariada. Los análisis estadísticos fueron realizados utilizando el SPSS Stadistics v24 (IBM Company). Resultados con un valor de significancia $(p)$ menor de 0,05 fueron considerados como estadísticamente significativos. Los valores perdidos se excluyeron del análisis correspondiente. 


\section{Resultados}

Este estudio caso-control, se centra en analizar diferentes variables de riesgo asociadas a tener cáncer de piel de tipo no-melanoma. Para ello, se realiza una comparación en la distribución de ciertos factores de riesgo conocido entre casos y controles (tabla 1). Los resultados muestran que los individuos con cáncer de células basales o escamosas suelen tener el pelo más claro que los controles $(p=0,001)$. Además, las personas con BCC o SCC presentan un mayor número de lesiones hiperpigmentadas benignas, tanto en el número de nevus $(p=0,013)$ como de lentigos solares $\left(p=3,77 \times 10^{-25}\right)$, respecto a los individuos que no han sufrido neoplasias cutáneas. Otro aspecto importante a tener en cuenta en la predisposición a desarrollar un cáncer cutáneo es la historia de exposición solar del individuo. Como muestran los resultados, los pacientes con BCC o SCC se han expuesto con mayor frecuencia a la radiación solar a lo largo de su vida $(p=0,019)$, seguramente debido a que han realizado más actividades al aire libre. Además, la edad de los pacientes con cáncer de piel es significativamente mayor que la de los individuos control $\left(p=1,77 \times 10^{-47}\right)$.

Además de los factores anteriormente analizados, la predisposición a cáncer de piel está influenciada por factores de carácter genético. Así pues, en este trabajo se analizó si había diferencias en el genotipo de ciertos genes clave en la síntesis de melanina entre los individuos con cáncer de piel y los individuos control (tabla 2). Los resultados muestran que los pacientes con BCC o SCC suelen ser portadores de variantes de pérdida de función en el gen $M C 1 R$ (gen clave en la capacidad de respuesta a la luz UV y directamente asociado a la predisposición al cáncer de piel), mientras que un menor porcentaje de individuos control eran portadores de alelos $\mathrm{R}$ $(p=0,019)$. Asimismo, un mayor porcentaje de individuos con cáncer de piel de tipo nomelanoma eran portadores del alelo ancestral A (asociado a rasgos de pigmentación más claros) del polimorfismo localizado en el gen ASIP (rs4911442), en comparación con los individuos control $\left(p=8,70 \times 10^{-4}\right)$.

Debido a que los individuos con BCC o SCC tenían una media de edad significativamente superior a los individuos control, se decidió repetir los análisis de asociación de variables de riesgo de cáncer cutáneo ajustándolos por la edad. De esta forma confirmamos que la asociación de los factores de riesgo analizados en este estudio a padecer cáncer cutáneo es independiente de la edad, posible variable de confusión en este estudio según el análisis descriptivo inicial. Este análisis únicamente se realizó para las variables anteriormente asociadas significativamente al riesgo de padecer cáncer de piel no-melanoma (tabla 3). Los resultados sugieren que, al ajustar por la edad de los individuos, tanto tener el color de pelo claro $(p=0,045)$ como tener un elevado número de lentigos solares $(p=0,009)$ son factores de riesgo importante en la aparición de cáncer de piel. Aunque la genética del individuo no parece tener un papel relevante al ajustar por la edad, se observa una tendencia a que ser portador de alelos $\mathrm{R}$ incrementa considerablemente el riesgo de padecer cáncer cutáneo $(\mathrm{OR}=7,03)$. 
Tabla 1

Distribución de las diferentes variables fenotípicas en casos y controles

\begin{tabular}{|c|c|c|c|}
\hline Característica fenotípica & Casos $(\mathrm{N}=56)$ & Controles $(\mathrm{N}=100)$ & p \\
\hline $\begin{array}{l}\text { Sexo } \\
\text { Hombre } \\
\text { Mujer }\end{array}$ & $\begin{array}{l}22(39,3 \%) \\
34(60,7 \%)\end{array}$ & $\begin{array}{l}38(38,0 \%) \\
62(62,0 \%)\end{array}$ & 0,874 \\
\hline $\begin{array}{l}\text { Color de ojos } \\
\text { Negro/Castaño } \\
\text { Verde } \\
\text { Azul/Gris }\end{array}$ & $\begin{array}{c}32(59,3 \%) \\
8(14,8 \%) \\
14(25,9 \%)\end{array}$ & $\begin{array}{l}66(66,0 \%) \\
20(20,0 \%) \\
14(14,0 \%)\end{array}$ & 0,173 \\
\hline $\begin{array}{l}\text { Color de pelo } \\
\text { Oscuro (Negro/Castaño oscuro) } \\
\text { Claro (Rubio/Castaño claro/Rojo) }\end{array}$ & $\begin{array}{l}24(44,4 \%) \\
30(55,6 \%)\end{array}$ & $\begin{array}{l}71(71,0 \%) \\
29(29,0 \%)\end{array}$ & 0,001 \\
\hline $\begin{array}{l}\text { Fototipo cutáneo según Fitzpatrick } \\
\text { Alto (III/IV) } \\
\text { Bajo (I/II) }\end{array}$ & $\begin{array}{l}33(60,0 \%) \\
22(40,0 \%)\end{array}$ & $\begin{array}{l}75(75,0 \%) \\
25(25,0 \%)\end{array}$ & 0,052 \\
\hline $\begin{array}{l}\text { Número de nevus } \\
\quad<50 \\
>50\end{array}$ & $\begin{array}{c}46(93,9 \%) \\
3(6,1 \%)\end{array}$ & $\begin{array}{l}76(77,6 \%) \\
22(22,4 \%)\end{array}$ & 0,013 \\
\hline $\begin{array}{l}\text { Quemaduras en la infancia } \\
\text { No } \\
\text { Pocas } \\
\text { Muchas } \\
\end{array}$ & $\begin{array}{l}24(47,1 \%) \\
19(37,4 \%) \\
8(15,7 \%)\end{array}$ & $\begin{array}{l}35(36,8 \%) \\
46(48,4 \%) \\
14(14,7 \%)\end{array}$ & 0,405 \\
\hline $\begin{array}{l}\text { Lentigos } \\
\text { No } \\
\text { Pocos } \\
\text { Muchos } \\
\end{array}$ & $\begin{array}{c}3(5,6 \%) \\
34(63,0 \%) \\
17(31,5 \%)\end{array}$ & $\begin{array}{c}91(92,9 \%) \\
5(5,1 \%) \\
2(2,0 \%) \\
\end{array}$ & $3,77 \times 10^{-25}$ \\
\hline $\begin{array}{c}\text { Historia exposición solar } \\
\text { No fotoexpuesto } \\
\text { Ocasionalmente } \\
\text { Habitualmente }\end{array}$ & $\begin{array}{l}6(11,3 \%) \\
18(34,0 \%) \\
29(54,7 \%)\end{array}$ & $\begin{array}{c}3(3,1 \%) \\
53(54,1 \%) \\
42(42,9 \%)\end{array}$ & 0,019 \\
\hline $\begin{array}{l}\text { Edad } \\
\quad \text { Media } \pm \text { Desviación típica }\end{array}$ & $72,54 \pm 11,56$ & $28,88 \pm 12,35$ & $1,77 \times 10^{-47}$ \\
\hline
\end{tabular}

Las variables cualitativas se presentan como número de individuos y el porcentaje de individuos por grupo respecto del total, mientras que las variables cuantitativas se presentan como media y desviación típica. Los resultados resaltados en negrita son significativamente diferentes entre grupos.

TABLA 2

Distribución de los genotipos encontrados en genes relacionados con pigmentación y sensibilidad solar en casos y controles

\begin{tabular}{|c|c|c|c|}
\hline Genotipo & Casos $(\mathrm{N}=56)$ & Controles $(\mathrm{N}=100)$ & $\mathbf{p}$ \\
\hline MC1R (variantes R) & & & 0,019 \\
\hline No portador de alelo $\mathrm{R}$ & $41(74,5 \%)$ & $83(89,2 \%)$ & \\
\hline Portador de alelo R & $14(25,5 \%)$ & $10(10,8 \%)$ & \\
\hline ASIP (rs4911442) & & & $8,7 \times 10^{-4}$ \\
\hline AA & $38(95,0 \%)$ & $64(64,0 \%)$ & \\
\hline$A G$ & $2(5,0 \%)$ & $25(25,0 \%)$ & \\
\hline GG & $0(0 \%)$ & $11(11,0 \%)$ & \\
\hline BCN2 (rs2153271) & & & 0,865 \\
\hline $\mathrm{CC}$ & $7(17,5 \%)$ & $14(14,0 \%)$ & \\
\hline CT & $15(37,5 \%)$ & $38(38,0 \%)$ & \\
\hline TT & $18(45,0 \%)$ & $48(48,0 \%)$ & \\
\hline IRF4 (rs12203592) & & & 0,844 \\
\hline CC & $27(67,5 \%)$ & $71(71,0 \%)$ & \\
\hline $\mathrm{CT}$ & $11(27,5 \%)$ & $23(23,0 \%)$ & \\
\hline TT & $2(5,0 \%)$ & $6(6,0 \%)$ & \\
\hline
\end{tabular}


Las variables cualitativas se presentan como número de individuos y el porcentaje de individuos por grupo respecto al total. Los resultados resaltados en negrita son significativamente diferentes entre grupos.

TABLA 3

Asociación de diferentes factores de riesgo de BCC o SCC ajustados por la edad

\begin{tabular}{lcc}
\hline \multicolumn{1}{c}{ Factor de riesgo } & $\mathbf{p}$ & OR $\mathbf{( 9 5} \%$ IC) \\
\hline Color de pelo claro & $\mathbf{0 , 0 4 5}$ & $5,59[1,04-29,94]$ \\
\hline $\mathbf{5 0}$ nevus & 0,560 & $1,90[0,22-16,09]$ \\
\hline Presencia de lentigos solares & $\mathbf{0 , 0 0 9}$ & $5,07[1,50-17,13]$ \\
\hline Frecuente exposición solar & 0,634 & $0,72[0,19-2,76]$ \\
\hline Alelo R en $\mathbf{M C 1 R}$ & 0,092 & $7,03[0,73-67,98]$ \\
\hline rs4911442*G en $\boldsymbol{A S I P}$ & 0,486 & $0,55[0,11-2,91]$ \\
\hline
\end{tabular}

El riesgo relativo se expresa como odds ratio (OR) y su intervalo de confianza (IC). Los resultados resaltados en negrita son significativamente diferentes entre grupos.

\section{Discusión y conclusiones}

Debido al incremento exponencial de la incidencia de cáncer de piel en todo el mundo, y especialmente en poblaciones de origen europeo, este estudio se centra en analizar las variables de riesgo asociadas a desarrollar un cáncer de células basales y escamosas en una población del Mediterráneo. El interés de estudiar esta población del sur de Europa radica en la falta de estudios en este campo llevados a cabo en estas poblaciones, ya que la mayoría de ellos se centra en estudiar poblaciones del norte de Europa que tienen fenotipos pigmentarios de mayor riesgo como el color de piel pálido y la incapacidad de broncearse tras exponerse al sol. Sin embargo, los individuos de origen mediterráneo suelen estar expuestos en mayor medida a la radiación UV ya que, debido a la buena climatología, suelen realizar actividades al aire libre prácticamente a diario. Sin embargo, menos de la mitad de la población adulta utiliza protección solar siempre para protegerse de los efectos nocivos del sol. Este hecho, junto con que un elevado porcentaje de individuos tiene la piel relativamente clara, hace que el cáncer de piel sea una enfermedad de gran importancia en nuestra región.

Como se ha comentado en la introducción, los rasgos de pigmentación humana y la sensibilidad solar son clave en el desarrollo de un cáncer de piel. Estas características fenotípicas están a su vez determinadas por variantes genéticas localizadas en genes involucrados de la síntesis de melanina (Liu, Wen y Kayser 2013). La acumulación de melanina por los queratinocitos puede ser de gran importancia en la aparición de un cáncer de piel de origen queratinocítico. Nuestros resultados confirman estudios previos donde se pone de manifiesto que los individuos que desarrollan un cáncer cutáneo suelen tener variantes genéticas asociadas a rasgos de pigmentación de riesgo, como son color de piel claro sin capacidad de broncearse, ojos claros, pelo claro y pecas (Scherer y Kumar 2010). Así pues, los resultados indican que ser portador de variantes de pérdida de función en el gen $M C 1 R$ aumenta el riesgo de padecer cáncer de células basales y escamosas. Las variantes $R$ de $M C 1 R$ limitan la síntesis de eumelanina, pigmento presente en la piel que absorbe los rayos UV para evitar que se dañe el ADN de las células de la piel. Según nuestros resultados, se 
observa una tendencia a presentar un mayor riesgo de padecer cáncer cutáneo no melanocítico en individuos portadores de alguna variante $R$, aunque dicha tendencia no es significativa al ajustar por la edad, seguramente por el poco número de individuos portadores de alelos $\mathrm{R}$ en la población de estudio. Esta tendencia observada concuerda con estudios previos que indican que el genotipo $M C 1 R$ es clave en la predisposición de un individuo a padecer cáncer de piel de tipo no-melanoma (Bastiaens et al. 2001). Los otros polimorfismos analizados en este estudio (localizados en tres genes relacionados con la pigmentación cutánea: ASIP, BCN2 e IRF4) no parecen tener un papel transcendental en el riesgo de BCC o SCC, seguramente porque el papel que juega la acumulación de radiación UV en la piel es fun damental en este tipo de cánceres cutáneos.

De la misma forma, los resultados de este estudio confirman la relación directa entre los rasgos de pigmentación humana y el riesgo de padecer cáncer cutáneo de tipo nomelanoma (basocelular o espinocelular). Aunque nuestros resultados no muestran una asociación entre el fototipo cutáneo y padecer un BCC o SCC, estudios previos han demostrado que aquellos individuos con un fototipo cutáneo bajo (I-II) tienen un mayor riesgo de cáncer de piel de tipo no-melanoma (Bastiaens et al. 2001). Esta asociación directa también es patente en el riesgo de melanoma, ya que los individuos que desarrollan un melanoma suelen tener una menor capacidad de bronceado y una tendencia a quemarse tras exponerse al sol (Fernandez et al. 2009).

Como se ha comentado en la introducción, la exposición solar prolongada es vital en el desarrollo de un carcinoma basocelular o espinocelular, ya que son lesiones cutáneas que suelen aparecer en áreas del cuerpo expuestas de forma habitual a la luz solar. Así pues, se observa que personas que se exponen de forma frecuente al sol tienen mayor riesgo de padecer un cáncer de piel no-melanoma. Además, la existencia de quemaduras en la infancia no parece estar relacionada con el aumento de riesgo de padecer BCC o SCC. Las quemaduras solares suelen estar relacionadas con una exposición solar más aguda e intensa relacionada comúnmente con periodos vacacionales y no con una exposición continuada típicamente relacionada con el cáncer de piel no-melanoma (Fernandez et al. 2009).

A pesar del incremento de la incidencia en jóvenes, el cáncer de piel no-melanoma es más frecuente en personas mayores que han estado expuestas al sol de forma intensa y prolongada por razones socioculturales durante un elevado periodo de tiempo. Nuestros resultados muestran que los pacientes con BCC o SCC tienen una media de edad significativamente mayor que los individuos control. Esta exposición continuada también concuerda con el hecho de que los individuos con BCC o SCC presentan una frecuencia mayor de lesiones hiperpigmentadas benignas que los controles. No obstante, esta diferencia de edad podría confundir los resultados de asociación observados en el resto de variables estudiadas. La mejor solución a dicho problema hubiera sido tener individuos control de edad avanzada. Sin embargo, debido a que los participantes del estudio se recogen por los especialistas en dermatología, la gente de avanzada edad no suele acudir a consulta a no ser que tengan problemas graves en la piel. Esto puede provocar un sesgo por la edad entre controles y casos. De ahí la necesidad de ajustar los análisis de asociación por la edad y confirmar la independencia de las asociaciones encontradas inicialmente.

Este estudio confirma la importancia de la exposición solar de forma continuada en la aparición del cáncer cutáneo de tipo no-melanoma (BCC y SCC), ya que encontramos una fuerte correlación con la edad y asimismo con la historia de exposición solar y la presencia de lesiones hiperpigmentadas benignas. Además, este riesgo parece estar influenciado por el genotipo en MC1R, gen clave en la respuesta a la radiación UV. 
La prevención y la detección precoz, así como una vigilancia especial de la población con mayor susceptibilidad para desarrollar un carcinoma cutáneo, son claves en la curación del cáncer de piel. Conocer los factores de riesgo en cada población permitiría ayudar a los especialistas en el diseño de campañas de prevención y seguimiento de pacientes según el riesgo individual de cada paciente.

\section{Referencias bibliográficas}

Apalla, Zoe, Dorothée Nashan, Richard Weller y Xavier Castellsagué. 2017. «Skin Cancer: Epidemiology, Disease Burden, Pathophysiology, Diagnosis, and Therapeutic Approaches». Dermatology and Therapy, 7(1): 5-19.

Bastiaens, Maarten, Jeannet ter Huurne, Christine Kielich, Nelleke Gruis, Rudi Westendorp, Bert Jan Vermeer y Jan Nico Bouwes Bavinck. 2001. «Melanocortin-1 Receptor Gene Variants Determine the Risk of Nonmelanoma Skin Cancer Independently of Fair Skin and Red Hair». American Journal of Human Genetics, 68(4): 884-894.

Beaumont, Kimberley, Richard Newton, Darren Smit, Helen Leonard, Jennifer Stow y Richard Sturm. 2005. "Altered cell surface expression of human MC1R variant receptor alleles associated with red hair and skin cancer risk». Human Molecular Genetics, 14(15): 2145-2154.

Fernandez, Lara, Roger Milne, Guillermo Pita, Uxua Floristan, Elena Sendagorta, Marta Feito, Jose Avilés, Manuel Martin-Gonzalez, Pablo Lázaro, Javier Benítez y Gloria Ribas. 2009. «Pigmentation-related genes and their implication in malignant melanoma susceptibility». Experimental Dermatology, 18(7): 634642.

Hernando, Bárbara, Elena Sanz-Page, Gerard Pitarch, Laura Mahiques, Francisca Valcuende-Cavero y Conrado Martinez-Cadenas. 2018a. "Genetic variants associated with skin photosensitivity in a southern European population from Spain». Photodermatology, Photoimmunology \& Photomedicine, 34(6): 415422.

Hernando, Bárbara, Maria Victoria Ibañez, Julio Alberto Deserio-Cuesta, Raquel SoriaNavarro, Inca Vilar-Sastre y Conrado Martinez-Cadenas. 2018b. "Genetic determinants of freckle occurrence in the Spanish population: Towards ephelides prediction from human DNA samples». Forensic Science International: Genetics, 33: 38-47.

Ibarrola-Villava, Maider, Lara Fernandez, Guillermo Pita, Jerónimo Bravo, Uxua Floristan, Elena Sendagorta, Marta Feito, Jose Avilés, Manuel Martin-Gonzalez, Pablo Lázaro, Javier Benítez y Gloria Ribas. 2010. "Genetic analysis of three important genes in pigmentation and melanoma susceptibility: CDKN2A, MC1R and HERC2/OCA2». Experimental Dermatology, 19(9): 836-844.

Jacobs, Leonie, Merel Hamer, David Gunn, Joris Deelen, Jaspal Lall, Dianavan Heemst, Hae Won Uh, Albert Hofman, André Uitterlinden, Christopher Griffiths, Marian Beekman Eline Slagboom, Manfred Kayser, Fan Liu y Tamar Nijsten. 2015. «A Genome-Wide Association Study Identifies the Skin Color Genes IRF4, MC1R, ASIP, and BNC2 Influencing Facial Pigmented Spots». The Journal of Investigative Dermatology, 135(7): 1735-1742.

Jacobs, Leonie, Andreas Wollstein, Oscar Lao, Albert Hofman, Caroline Klaver, André Uitterlinden, Tamar Nijsten, Manfred Kayser y Fan Liu. 2013. "Comprehensive candidate gene study highlights UGT1A and BNC2 as new genes determining continuous skin color variation in Europeans». Human Genetics 132(2): 147158.

Liu, Fan, Bei Wen y Manfred Kayser. 2013. «Colorful DNA polymorphisms in humans». Seminars in Cell \& Developmental Biology, 24(6-7): 562-575. 
Matichard, E., Patrice Verpillat, R. Meziani, Benedicte Gérard, Vicent Descamps, E. Legroux, M. Burnouf, G. Bertrand, Fabrice Bouscarat, A. Archimbaud, Catherine Picard-Dahan, L. Ollivaud, Nicole Basset-Seguin, Delphine Kerob, G. Lanternier, Celeste Lebbé, Béatrice Crickx, Bernard Grandchamp y Nadem Soufir. 2004. «Melanocortin 1 receptor (MC1R) gene variants may increase the risk of melanoma in France independently of clinical risk factors and UV exposure». Journal of Medical Genetics, 41(2): e13.

Scherer, Dominique y Rajiv Kumar. 2010. «Genetics of pigmentation in skin cancer - A review». Mutation Research/Reviews in Mutation Research, 705(2): 141-153.

$\mathrm{Wu}$, Xufeng y John Hammer. 2014. "Melanosome transfer: it is best to give and receive». Current Opinion in Cell Biology, 29: 1-7. 